Cahiers d'études italiennes

1 | 2004

NOVECENTO... E DINTORNI

Dire la guerre?

\title{
La dernière course de Milton furieux
}

Michèle Coury

\section{OpenEdition \\ Journals}

Édition électronique

URL : http://journals.openedition.org/cei/236

DOI : $10.4000 /$ cei.236

ISSN : 2260-779X

Éditeur

UGA Éditions/Université Grenoble Alpes

Édition imprimée

Date de publication : 15 novembre 2004

Pagination : 91-103

ISBN : 978-2-84310-057-4

ISSN : 1770-9571

Référence électronique

Michèle Coury, "La dernière course de Milton furieux », Cahiers d'études italiennes [En ligne], 1 | 2004, mis en ligne le 15 mai 2006, consulté le 27 mars 2021. URL : http://journals.openedition.org/cei/236 DOI : https://doi.org/10.4000/cei.236 


\title{
LA DERNIĖRE COURSE
}

DE MILTON FURIEUX

\author{
Michèle Coury \\ Université Stendhal-Grenoble 3
}

À l'origine de ma réflexion sur le roman Una questione privata, cette citation d'Italo Calvino extraite de la préface à l'édition de 1964 de Il sentiero dei nidi di ragno:

Una questione privata è costruito con la geometrica tensione d'un romanzo di follia amorosa e cavallereschi inseguimenti come l'Orlando Furioso e nello stesso tempo c'è la Resistenza proprio com'era, di dentro e di fuori, vera come mai era stata scritta [...] Ed è un libro di paesaggi, ed è un libro di figure rapide e tutte vive, ed è un libro di parole precise e vere. Ed è un libro assurdo, misterioso, in cui ciò che si insegue, si insegue per inseguire altro, e quest'altro per inseguire altro ancora e non si arriva al vero perché ${ }^{1}$.

On sait le goût de Calvino pour les récits d'inspiration chevaleresque où plusieurs fils s'entrelacent autour de chevaliers errants et où toute poursuite rebondit vers une autre poursuite. En outre, dans le titre posthume du roman, l'adjectif "privata» nous fait pressentir le caractère mystérieux de ladite question, au sein d'un roman sur la Résistance. L'adjectif est utilisé une fois par le protagoniste lui-même. À un partisan qu'il vient

1. Italo Calvino, Il sentiero dei nidi di ragno, nouvelle édition avec une préface de l'auteur, Torino, Einaudi, 1964. Citons en outre un passage de Orlando Furioso raccontato da Italo Calvino (Torino, Einaudi, 1970) qui met en évidence la parenté entre le chevalier et le partisan, tous deux en proie à une idée fixe, étrangère à leurs occupations guerrières: «L'armata di Carlo Magno è stretta d'assedio tra le mura di Parigi. La notte, le sentinelle scrutano dagli spalti i fuochi dell' accampamento saraceno che circonda da ogni lato la città. Ma non sono solo le sentinelle a vegliare. Sul suo letto da campo, Orlando continua a rivoltolarsi senza poter prendere sonno: la sua mente è occupata da un pensiero che ha insieme la fissità e l'irrequietezza d'un riflesso di luna sul mare increspato dalle onde. E' all'assedio, alle battaglie, alle fortune dell'esercito franco in pericolo, che egli pensa? No, egli è lontano dai pensieri che soli dovrebbero albergare nell'animo di un paladino suo pari, esempio di dedizione al dovere e castità. Appena le sue palpebre cedono fuggevolmente al sonno è sempre la medesima immagine che gli appare: Angelica. Egli non sa pensare ad altro, non sa far altro che smaniare perché l'ha perduta e non sa dove sia. [...] Sella il suo cavallo Brigliadoro, passa silenziosamente davanti alla tenda di suo zio Carlo Magno, e senza chiedergli permesso, di nascosto, esce da Parigi, alla ricerca d'Angelica». 


\section{MichèLE COURY}

de rencontrer par hasard et qui s'interroge sur le fait de le voir habillé en civil, Milton répond "Vengo da Santo Stefano, per una mia cosa privata $^{2} »$.

Comment se distingue cette question privée de ce qui lui fait fond, c'est-à-dire l'affaire collective, l'univers des partisans et plus largement encore la guerre elle-même qui est l'affaire de tous et pas seulement des partisans? Telle est la "question» justement et je rappellerai d'abord les différentes acceptions que le terme nous invite à prendre en considération: le sens générique d'affaire, de sujet (voir, sur ce point, la traduction $\mathrm{du}$ titre en français "une affaire personnelle ${ }^{3}$ ») ; l'interrogation; dans le sens plus étymologique de la quaestio, la recherche, la quête dont dépend le sens de l'existence; je rajouterai, sans trop forcer le sens que Milton se soumet lui-même au supplice de la question, à une torture qui, comme il le dit lui-même, le transperce de part en part comme une baïonnette ${ }^{4}$.

Ce qui scande le déroulement de cette question personnelle, ce sont les accélérations et les décélérations d'une course ininterrompue (et qui s'interrompra par force, au dernier chapitre, dans une sorte d'apothéose). Milton est un garçon qui n'arrête pas de courir d'une garnison à l'autre. On peut relever dans le roman de nombreuses expressions qui font référence à son pas naturellement ample et rapide mais que le déclenchement de l'idée fixe va considérablement accélérer ${ }^{5}$. Paradoxalement, c'est l'idée fixe, c'est-à-dire l'espace intérieur et secret de Milton qui est le moteur de toute l'action du roman et qui nous révèle réalistement, à nous lecteurs, des aspects de la vie des partisans.

Toutefois si ces deux espaces, celui de la question qui taraude et celui de la lutte au jour le jour des partisans, se mélangent dans le récit, il n’en va pas de même entre les personnages. "La question privée» ne sera jamais l'objet d'un dialogue ou d'un échange quelconque avec qui que ce soit, ni partisan, ni civil rencontré à la faveur des déplacements (si ce n'est au tout début, puisque c'est par le biais d'une conversation avec un tiers que commence toute l'histoire ${ }^{6}$ ).

2. Beppe Fenoglio, Una questione privata, Torino, Einaudi, 1986, p. 121. Les citations font référence à cette édition.

3. Beppe Fenoglio, Une affaire personnelle et autres récits, Paris, Gallimard, 1978.

4. Chap. XI, p. 140 : «Doveva sapere che quelle sue parole mi passavano da parte a parte come baionette."

5. Chap. I, p. 4: «Aveva gambe lunghe e magre, cavalline che gli consentivano un passo esteso, rapido e composto"; chap. II, p. $21:$ : Si avventò di corsa per il vialetto dei ciliegi, passando in tromba Ivan » ; chap. IV, p. 40 : «La sofferenza gli fece accelerare il passo ».

6. Alors qu'il n'a pu résister au fait de revoir la maison de Fulvia, la jeune fille dont il est amoureux et qui est partie à Turin depuis plus d'un an, le partisan Milton rencontre la gardienne, seule âme qui vive dans la maison désertée. Au détour de la conversation, la femme laisse entendre que Fulvia aurait entretenu une relation intime avec Giorgio, leur ami commun. 
Autour de la question d'ordre privé, se condense l'absolue solitude de Milton qui sera d'ailleurs exprimée de façon définitive au dernier chapitre. Il convient de préciser à ce point que les deux personnages directement concernés par la question personnelle de Milton c'est-à-dire, Fulvia, la jeune fille aimée et Giorgio l'ami-rival n'interviennent jamais directement dans le récit ${ }^{7}$. Ils appartiennent à l'univers mental de Milton et ne sont introduits dans le récit que selon deux modalités: celle, privilégiée, de la mémoire et celle de la lettre mentale.

Milton, auteur infatigable de lettres à Fulvia, du temps où ils étaient en relation, continue tout au long de sa course de s'adresser intérieurement à elle dans une ébauche de lettre incandescente.

La question privée, dès qu'elle commence à obséder Milton, après les révélations de la gardienne, le met en porte à faux, lui donne le sentiment d'être devenu indisponible pour le combat collectif.

Il fatto è che più niente m'importa. Di colpo, più niente. La guerra, la libertà, i compagni, i nemici. Solo più quella verità. [.....] Oggi era diventato indisponibile, di colpo, per mezza giornata, o una settimana, o un mese, fino a quando avesse saputo. (chap. III, p. 28 et 31)

L'obsession de Milton, très intériorisée, se traduit pour l'extérieur par une faille dans son comportement habituel. Si Milton ne communique en rien ce qui le hante, son comportement semble parfois étrange aux yeux de ses camarades et on observera la récurrence de termes comme "fou", «devenu fou» qui rendent compte de cette étrangeté pour autrui. De même les verbes "de-viare», "de-lirare» utilisés respectivement au premier et au dernier chapitre marquent étymologiquement cette excursion hors du chemin du devoir partisan: "pourquoi as-tu dévié?» demande le partisan Ivan, voyant que Milton, sans raison apparente, a changé d'itinéraire (chap. I, p. 3). «Stanotte ero pazzo, certo deliravo per la febbre.» (chap. XIII, p. 150). Rappelons qu'au premier chapitre, c'est bien parce qu'il dévie de son itinéraire programmé, ne pouvant résister à la tentation de revoir la maison de Fulvia, que Milton va rentrer dans l'engrenage dramatique de la recherche effrénée.

La question - recherche de Milton s'effectue à l'intérieur d'une structure circulaire (à la fin du récit, on revient au point de départ) selon une modalité d'opposition fini-infini, temps réel-temps mythique, dans un brouillage de la perception du temps ou des temps. Entre le début et la fin

7. C'est du moins le cas dans la troisième et dernière version de Una questione privata, celle qui a été publiée. Dans la première, Fulvia cherche à intervenir pour faire libérer Giorgio. Voir à ce propos Rosella Cuzzoni. "Le tre redazioni di Una questione privata» in AA.V. Atti del convegno nazionale di studi fenogliani (Alba, 7-8 Aprile 1973) in «Nuovi Argomenti», p. 35-36, 1973. 
du récit, quatre jours seulement se sont écoulés et pourtant il semble impossible de quantifier ce temps (de même qu'il s'avère difficile d'évaluer les distances parcourues par Milton alors que tout advient dans un périmètre réduit).

- D’une part, parce qu'à travers le travail obsessionnel de la mémoire, le temps semble se dilater. Un souvenir fait naître un autre souvenir...

- D'autre part, contenir le temps c'est fixer des termes à l'action, aux événements. " $\mathrm{E}$ ' solo di un termine che ha bisogno la povera gente" (chap. VIII, p. 78). «Quando finirà la guerra?» demandent plusieurs personnes à Milton. "Le jour viendra où...» les fascistes seront noyés dans un bain de sang, selon les uns, où l'espoir et la joie reviendront et où la jeunesse dansera à nouveau, selon les autres. Cette expression, "verrà quel giorno...", qui renvoie au terme de la fin de la guerre, c'est aussi le début d'un vers d'un poème anglais cher à Milton, de Robert Browning, sur lequel nous reviendrons plus loin et dont nous verrons qu'il constitue l'hypotexte du discours amoureux de Milton. Si l'on se rapporte au poème en question on constate qu'il est dit dans l'une des strophes à travers la traduction de Fenoglio lui-même, faite en 1951:

Ma quel tempo verrà - alla fine verrà / Quando, Evelyn Hope, mi domanderò cosa significarono [È] / Quel corpo e quell'anima così puri e felici; / [...] saprò [...] ciò che con me farai, alla fine, / Nella vita nuova venuta al posto dell'antica ${ }^{8}$.

À la première page du roman, quand Milton se retrouve face à la maison de Fulvia, avant que la gardienne ne lui révèle ce qui va le bouleverser et le précipiter dans sa "question personnelle», il s'interroge sur le futur de son amour et le situe par rapport à ce terme de la fin de la guerre.

Quando la rivedrò? Prima della fine della guerra è impossibile. Non è nemmeno augurabile. Ma il giorno stesso che la guerra finisce correrò a Torino a cercarla. E' lontana da me esattamente quanto la nostra vittoria. (chap. I, p. 3)

8. Voir à ce propos le document annexe de l'étude exhaustive suivante: Gabriele Pedullà, La strada più lunga. Sulle tracce di Beppe Fenoglio, Roma, Donzelli edit., 2001. La traduction du texte original de R. Browning est celle de B. Fenoglio, faite en mai 1951. Durant cette année-là, Beppe Fenoglio effectue une série de lectures de ses traductions d'auteurs anglais et américains (Eliot, Pound, Coleridge) dans le cadre des activités culturelles du Circolo Sociale où se retrouve la bourgeoisie d'Alba.

But the time will come, - at last it will

When, Evelyn Hope, what meant (I shall say)

In the lower earth, in the years long still,

That body and soul so pure and gay?

Why your hair was amber, I shall divine,

And your mouth of your own geranium's red -

And what you would do with me, in fine,

In the new life come in the old one's stead. 
Or c'est à ce terme de la fin de la guerre que la question personnelle, elle, va substituer le terme toujours déplacé d'une recherche sans fin qui finit par devenir à elle-même son propre objet. La question personnelle "quelle est la vérité? quand saurai-je la vérité?» recouvre la question collective "quand finira la guerre?».

L'objet de la recherche de Milton ne cesse de se démultiplier, en même temps qu'il court de-ci, de-là. Que recherche Milton? Milton recherche la vérité de la relation entre Fulvia et Giorgio. On sait que la vérité est un mot dangereux. Milton voudrait que ne subsistât aucun doute mais le lecteur, lui, peut se demander à juste titre en quoi subsiste le moindre doute dans les paroles de la gardienne qui sont bien assez claires.

Par ailleurs, il ne semble pas, à la lecture du texte, que Fulvia se soit jamais engagée de façon claire; elle cultive une ambiguïté, elle laisse entendre que, elle se reprend, elle parle de façon cryptée, en latin ${ }^{9}$. Milton serait donc à la recherche d'une absolue clarté qui, cependant, n'a jamais caractérisé sa relation avec Fulvia, bien au contraire. Alors peut-être est-il inversement à la recherche d'un doute qui mette à distance un impact trop dur avec l'aridité de la réalité. La manie de Milton est l'interprétation et sa véritable fureur, la fureur herméneutique: d'un côté créer du doute là où il n'y en a pas ou fort peu, de l'autre sur-interpréter, s'acharner à donner du sens à ce qui se tient dans les limbes de l'indéterminé. Il en a d'ailleurs l'intuition vers la fin du récit lorsqu'il pense «forse Fulvia mi ha fatto costruire tutto un mondo di amore su certe parole dette così per dire...» (chap. XI, p. 140).

Quoi qu'il en soit, au nom de la recherche de la vérité, Milton part à la recherche d'un Giorgio devenu nécessaire, objet secondaire d'une quête qui n'en finit pas de rebondir vers d'autres objets dérivés. Giorgio ayant été fait prisonnier par les fascistes, à la faveur d'un brouillard emblématique, Milton part à la recherche d'un fasciste à échanger contre lui. Contraint de tirer sur le prisonnier fasciste au moment où celui-ci tente de lui échapper, Milton voit donc s'évanouir cette possibilité de retrouver Giorgio et de savoir la vérité. (Mais pourquoi penser que Giorgio aurait dit forcément la vérité?) Au dernier chapitre, Milton décide de retourner voir la gardienne pour réentendre l'histoire une deuxième fois et chercher à savoir directement la vérité. C'est à cette occasion qu'il tombera sur une patrouille de soldats fascistes, qu'il trouvera justement ceux qu'il n'a pas cherchés.

9. «Ma più tardi disse, piano ma che lui sentisse sicuramente: "Hieme et aestate, prope et procul, usque dum vivam...”" chap. I, p. 6. 
La phrase clef du roman est celle qu'il formule après avoir tué le soldat fasciste «Ma domani, come passerò domani senza il programma nemmeno di cercare?» (chap. XI, p. 132).

Au moment même où il s'apprête à retourner voir la gardienne, au dernier chapitre, il hésite, il est assailli par le doute sur le bien fondé de cette ultime démarche, alors même que s’affirme en lui la désespérante évidence d'une relation bel et bien consommée entre Giorgio et Fulvia.

Non c'è nulla da chiarire, da approfondire, da salvare. Non ci sono dubbi. Ci vado, ci vado ugualmente. Non saprei proprio che altro fare e non posso stare senza fare niente. (Chap. XIII, p. 150)

Le fait que tout "agir" se condense autour de ce programme de chercher sans relâche, infiniment, annule de fait toute possibilité de réponse et, selon nous, accrédite a contrario, le parti pris que le récit, lui, doit bien finir ainsi, c'est-à-dire sans réponse.

Comme l'écrit Dante Isella, dans son édition critique des œuvres de Fenoglio:

Ma il tormentoso interrogativo di Milton, la sua ricerca della verità, non sono forse dobbiamo chiederci, un interrogativo e una ricerca che non possono ottenere risposta ${ }^{10}$.

Si comme nous l'avons dit, la question du but collectif est occultée par le but à atteindre dans l'interrogation personnelle, c'est que le temps qui compte, qui détient la clef, est celui "d'avant», qui resurgit, par bribes, dans la mémoire. Le temps d'avant quoi?

Globalement, le temps d'avant les révélations de la gardienne, qui luimême se décompose en deux époques:

- le temps de la guerre civile qui a commencé à l'automne 1943;

- mais surtout le temps d'avant la guerre civile, d'avant la Résistance quand Milton, Giorgio et Fulvia se retrouvaient dans la maison des collines.

C'est ce temps-là qui va se faire réservoir de souvenirs à la fois lumineux et déchirants, alimentant le feu de l'obsession. Un des moments forts de ce "temps d'avant» se situe durant le printemps 1942: Fulvia grimpe dans un des cerisiers de son jardin et offre à Milton les cerises les plus glorieusement mûres (chap. I, p. 5). En ce temps-là, Milton ne s'appelait pas encore Milton et à aucun moment son nom civil ne sera précisé dans le cours du récit. Au premier chapitre du roman qui évoque le tout début de sa relation avec Fulvia, il est appelé simplement «le futur Milton " c'est-àdire celui dont le nom s'inscrira dans le rappel implicite d'un Paradise

10. Beppe Fenoglio, Romanzi e racconti, a c. di Dante Isella, Einaudi-Gallimard, 1992, p. 1640. 
Lost $^{11}$. Quand ce temps passé dans le jardin de la maison de Fulvia finit-il?

Durant l'été 1943, Milton achève son service militaire. Il n'est pas à Alba. Ce même été, dans la maison sur les collines, aux dires de la gardienne, Fulvia répondant à la séduction de Giorgio aurait peut-être «fait le mal» (chap. II, p. 19). Fulvia abandonne précisément la maison, le 12 septembre, quelques jours après l'armistice pour retourner à Turin. À cette même date, Milton se souvient s'être caché dans les sordides toilettes de la gare de Livourne avant de trouver le moyen de remonter vers le Piémont, en l'occurrence caché par un cheminot dans le wagon à charbon ${ }^{12}$ (chap. II, p. 20). On peut dire que Milton entre dans la Résistance comme il entre dans l'Absence de Fulvia, sur une Chute. (Entre temps, il se peut que le mal ait été consommé mais il ne le sait pas encore.)

Il nous semble à présent opportun de revenir sur la structure circulaire du récit en reliant la course furieuse de Milton au dernier chapitre et le temps d'avant, évoqué essentiellement dans les premiers chapitres.

Au dernier chapitre, on revient au lieu du chapitre premier, c'est-à-dire la maison de Fulvia. Dans un cas comme dans l'autre, Milton ne peut résister à cette tentation qui, une première fois, après les révélations de la gardienne, le détruira psychologiquement et une deuxième fois l'exposera au tir des soldats fascistes. Cette structure circulaire, par delà la recherche qui rebondit sans cesse et dont on a vu qu'elle était caractérisée par l'impossibilité de rejoindre et de posséder son objet, semble refermer un destin de mort, ou plus exactement un destin où la mort donne le sens: qu'elle soit la mort, impitoyablement menaçante et réelle de l'expérience partisane ou la mort idéalisée, cachée dans les replis de nombreuses références textuelles, dans un appel à la Vérité d'une autre Vie (c'est-à-dire le déni de la mort.)

$\mathrm{Au}$ début du $\mathrm{I}^{\mathrm{er}}$ chapitre, lorsque Milton arrive devant la maison de Fulvia et avant qu'il ne rencontre la gardienne, la demeure lui apparait encore blanche, sans taches, ni traces de fumée. Elle a gardé son éclat bien que les fenêtres soient refermées au loquet sur l'inviolabilité de Fulvia et de sa beauté. Au chapitre XIII, Milton revient vers la maison sous une pluie battante mais cette fois-ci, elle lui apparaît bien différente:

11. Le nom de guerre du jeune partisan féru de littérature anglaise et américaine est évidemment emprunté au poète anglais atteint de cécité John Milton (1608-1674). Rappelons que son œuvre majeure Paradise Lost (1667) évoque la colère des anges rebelles et leur chute en Enfer, la séduction d'Ěve par Satan principal acteur de ce drame. Le fruit qu'il invite à cueillir représente le serment qui lie l'humanité au Démon.

12. Chap. II, p. 20. «E lui, lui dove era il dodici settembre 1943? [...] A Livorno, asserragliato nei cessi della stazione, digiuno da tre giorni, miserabilmente vestito di panni d'accatto. Sul punto di svenire per l'inedia e le esalazioni della latrina si era affacciato sul corridoio e aveva cozzato in quel macchinista che si stava abbottonando la brachetta. [...] Si parte tra mezz'ora, ma ti voglio nascondere subito nella carbonaia. Mica te ne frega di sembrare poi uno spazzacamino?». 
fantomatica, velata dalle cortine della pioggia che concorrevano a sfigurarla. Egli la vide decisamente brutta, gravemente deteriorata e corrotta, quasi fosse decaduta di un secolo in quattro giorni. (chap. XIII, p. 150)

Cette maison telle qu'elle apparaît au Ir chapitre encore blanche et belle, bien que désertée et barricadée, est un temple sacré qui abrite le temps d'avant la Résistance, la relation Milton-Fulvia mais aussi la relation triangulaire Milton-Fulvia-Giorgio.

Rappelons ce qui caractérise cette relation appartenant au temps d'avant. Une image: Giorgio et Fulvia dansent, Milton regarde et met les disques. D'un côté, les nantis de l'apparence, l'aisance financière, l'aisance du corps, la beauté, la séduction, de l'autre, celui qui doit compenser la gaucherie et le physique ingrat, par la beauté des yeux: selon un cliché dualiste, la beauté des yeux révèle la "vraie» beauté, celle de l'âme. L'amour de Milton pour Fulvia est, on l'a vu, un amour littéraire qui passe par "les lettres", c'est-à-dire la littérature et les lettres que l'on envoie. Ainsi écrit-il au début de leur relation «Fulvia splendore. Fulvia dannazione» avec ce goût pétrarquisant de l'antithèse qui relie son expression à toute une tradition courtoise et post-courtoise où la Dame est maître de la mort et du salut ${ }^{13}$. Mais dans sa volonté de plaire à Fulvia, Milton est avant tout un traducteur, un traducteur orienté et partial qui aime isoler des textes qu'il traduit, des citations pertinentes à son projet de transfiguration idéalisante de la bien-aimée. Ainsi "Oh my love oh my lost love Morella» est-elle une citation hybride de deux textes d'Edgard Poe, Morella et Ligeia à travers laquelle Milton fait implicitement une double

13. Précisons que la référence à Pétrarque n'est pas explicite, dans le roman. Toutefois, comme il sera d'ailleurs précisé plus loin dans cette étude, au début du roman, lorsque Milton se rend sur le seuil de la maison de Fulvia, il "dédie» à cette dernière sa vie de combattant sous forme d'antithèses. Si le propos suit le tracé du poème de Robert Browning, l'expression peut éveiller des réminiscences pétrarquiennes. Il nous plaît de citer ce sonnet de Pétrarque que les propos de Milton peuvent tout à fait rappeler.

Pace non trovo e non ò da far guerra,

E temo e spero; ed ardo e son un ghiaccio;

E volo sopra'l cielo e giaccio in terra;

E nulla stringo, e tutto'l mondo abbraccio.

Tal m’à in pregion, che non m'apre né serra,

Né per suo mi riten né scioglie il laccio;

E non m'ancide Amore e non mi sferra,

Né mi vuol vivo né mi trae d'impaccio.

Veggio senza occhi e non ò lingua e grido;

E bramo di perir e cheggio aita;

Ed ò in odio me stesso ed amo altrui.

Pascomi di dolor, piangendo rido;

Egualmente mi spiace morte e vita:

In questo stato son, Donna, per voi.

Sonnet CXXXIV 
référence à celle qui renaît du royaume de la mort, pour mourir encore et dit au narrateur «je vais mourir, cependant je vivrai ${ }^{14}$ ».

Milton fait endosser par les auteurs qu'il traduit un discours qu'il ne prend que partiellement à son compte (puisqu'il n'en est que le traducteur) mais qui est destiné à injecter de façon subliminale, des rayons idéalisants et mortuaires qui pourraient faire en sorte que Fulvia devienne ce qu'il veut qu'elle soit. Après quelque temps de fréquentation, Fulvia cesse de lire les livres qu'elle avait choisis, elle, (Proust, Schnitzler) et se contente de poésies et de récits que Milton lui traduit sans relâche. Elle tente d'échapper à cette influence dont elle est consciente:

Basta, non mi parlare più. Mi fai piangere. Peggio che triste, sei tetro. E io non voglio diventare triste come te. Io sono bella e allegra. Lo ero. (chap. II, p. 13)

L'emprise pourrait être réciproque. Sa beauté le subjugue mais lui, de son côté, voudrait faire d'elle sa chose littéraire, sa création. D'ailleurs, sa dernière lettre mentale, au dernier chapitre, au moment où il court sous les balles ennemies, ne sera-t-elle pas?

Ti sto pensando anche ora, anche in queste condizioni, sto pensando a te. Lo sai che se cesso di pensarti, tu muori istantaneamente? (chap. XIII, p. 150)

Dans un registre plus humble, le premier disque qu'il lui offre et lui traduit et qui devient le leitmotiv de leur histoire est la chanson américaine Over the rainbow (à laquelle Fenoglio avait pensé, dans un premier temps, pour être le titre du roman). Milton continue de la fredonner à certains moments, durant sa recherche.

Somewhere over the rainbow / Skies are blu / And dreams that you dare to dream really do come true / That's where you'll find me / Somewhere over the rainbow, bluebirds fly / Why oh why can't I?

Dans le corpus, non laissé au hasard, de textes ou chansons que Milton choisit de traduire, très récupérables dans sa fiction idéalisante, une place à part revient au texte de Robert Browning, Evelyn Hope dont il a déjà été fait mention plus haut, pas seulement parce que c'est la première poésie que le jeune homme traduit pour Fulvia ("Ma sei un vero dio» s'écrie-telle après avoir lu la traduction), mais parce qu'il s'agit de la mort d'une jeune fille de seize ans, l'âge de Fulvia justement à ce moment là. "Beautiful Evelyn Hope is dead / Sit and watch by her side an hour ${ }^{15}$ " cite justement Milton (chap. I, p. 8).

14. "La volta dopo le portò un racconto di Poe. "Di che parla?" "Of my love, of my lost love Morella". "Lo leggerò stanotte". "Io l'ho tradotto in due notti" ". Voir Gabriele Pedullà, op. cit., p. 26. 15. Robert Browning, "Evelyn Hope», in Men and Women (1855). 
Il est intéressant de rappeler certains éléments de la vie du poète victorien Robert Browning, qui éclairent indirectement le texte Una questione privata même si ni Milton ni l'auteur Fenoglio ne mentionnent directement son nom. La vie de Browning est l'histoire exemplaire d'un amour absolu, idéal, vécu avec sa propre épouse, la poétesse Elisabeth Barrett; il commence par un échange de lettres, se poursuit dans le ravissement puisque le poète enlève la jeune fille à sa famille pour l'emmener en Italie. Ils y vivent quinze années d'un bonheur auquel seule la mort de la bienaimée viendra mettre un terme. Dans sa vieillesse, il écrit attendre de la rejoindre, elle, mi-ange, mi-oiseau dans l'espoir que, malgré l'éloignement et l'ombre, ce qui fut puisse être à nouveau ${ }^{16}$.

S'il convient de s'arrêter sur la poésie Evelyn Hope c'est qu'elle sert de tracé, du reste non énoncé par l'auteur, à la sombre déambulation de Milton parmi les pièces et les meubles de la maison de Fulvia au début du roman. D’un côté, le poète anglais évoque les objets de la jeune fille morte:

La bella Evelyn è morta! / Siedi e vegliala un'ora. / Quello è il suo scaffale, questo il suo letto ; / Voleva spetalare quel geranio / Che comincia a morire, anch'esso, nel cristallo / Poco è stato ancora, mi pare. / Le imposte chiuse, non luce che filtri / Salvo due lungi raggi per la fessura del cardine 17

Traduction B. Fenoglio.

De l'autre, Milton erre dans le salon non pas d'une morte, mais d'une absente, alors qu'une lumière spectrale tombe sur la pièce. La gardienne ne fait-elle pas remarquer qu'on a l'impression de rentrer dans une tombe? Sur le pas de la maison de Fulvia, avant que la gardienne n'ait fait ses révélations, Milton a déjà synthétisé sa propre vie de partisan, en quelques fulgurantes antithèses qui, outre les réminiscences pétrarquisantes, trahissent d'indéniables similitudes avec une des strophes du poème anglais :

16. Voir Robert Browning, The ring and the book (1868). Voir Daniel Karlin, The courtship of R. Browning and Elisabeth Barrett, Oxford University Press, 1985.

17. Texte original de Robert Browning

Beautiful Evelyn Hope is dead

Sit and watch by her side an hour.

That is her book-shelf, this is her bed;

She plucked that piece of geranium-flower,

Beginning to die too, in the glass;

Little has yet been changed, I think:

The shutters are shut, no light may pass

Save two long rays thro'the hinge's chink. 
Ho fatto tanto, ho camminato tanto sono scappato e ho inseguito. Mi sono sentito vivo come mai e mi sono visto morto. Ho riso e ho pianto. Ho ucciso un uomo a caldo. Ne ho visti uccidere, a freddo, moltissimi. Ma io sono sempre lo stesso. (chap. I, p. 9) ${ }^{18}$

Au dernier chapitre, Milton n'en finit pas de chuter dans la boue «son fatto di fango dentro e fuori» (chap. XIII, p. 150). Il devient lui-même un oxymoron "uno spettro fangoso" traduisant la dualité de sa course à la fois concrète et irréelle.

Contrastant avec la pesanteur du corps, avec cette matérialité qui condense le lot du partisan - le froid, la boue, la douleur physique - se trace un mouvement d'oiseau prenant son envol (over the rainbow). "Correva ma senza contatto con la terra» (chap. XIII, p. 154).

Portant le mouvement du personnage, le texte lui-même décolle d'un plan réaliste à un plan allegorico-symbolique. Le personnage et le texte sont en suspension entre la terre et le ciel, entre la réalité crûe du combattant pris en point de mire et cette immatérialité spectrale qui appartient, comme on l'a vu, à tout un fond de réminiscences poétiques qui idéalisent la mort.

Aveva bisogno di veder gente e d'esser visto per convincersi che era vivo, non uno spirito che aliava nell'aria, in attesa di incappare nelle reti degli angeli (chap. XIII, p. 154).

Milton, sur le point d'être pris dans les filets des anges et, pourrait-on ajouter, dans les filets de tous ces spectres féminins qui vont et viennent entre la vie et la mort et qui appartiennent à son imaginaire de jeunesse.

Il est intéressant de constater que cette course sur laquelle se clôt le roman est une reprise d'un chapitre de Il Partigiano Johnny où Johnny s'écroule, mais non point pour mourir ("vide nero e crollò » ${ }^{19}$ ). Cela a pu accréditer l'idée que le roman Una questione privata n'est pas fini et que le verbe "crollò ", dernier mot du texte ne signifie pas forcément la mort de Milton. Toutefois, si on fait une étude comparative très précise des deux textes très proches, on remarquera combien Fenoglio insiste davantage dans celui qui nous occupe ici, sur l'état de suspension, sur la tentation

18. Ibid., chap. I, p. 9.

Texte de Robert Browning «Evelyn Hope» et traduction de Beppe Fenoglio

I have lived (I shall say) so much since then, Given up myself so many times,

Gained me the gains of various men,

Ransaked the ages, spoiled the climes;

Yet one thing, one, in my soul's full scope,

Either I missed or itself missed me:

And I want and find you, Evelyn Hope!

What is the issue? let us see!

Voir ce qui a été dit note 13 de cette étude.

19. Beppe Fenoglio, Il partigiano Johnny, a c. di Dante Isella, op. cit, p. 779.
Dirò : ho vissuto così tanto da allora, Mi sono arreso così tante volte,

Arrogato le conquiste di vari uomini, Ho frugato le età ed assaggiato i climi;

Pure una cosa, una, in tutto il giro dell'anima mia, Mi è mancata od io sono ad essa mancato. Ed io ti voglio e ti troverò, Evelyn Hope! Come finirà? Vedremo. 
d'une immatérialité spectrale qui retombe vers la terre. La répétition anaphorique du verbe "correva» nous indique que cette course à l'aveugle, justement vers on ne sait quel but, représente le point culminant, le pointlimite de cette question mise en branle pour accéder à un but inatteignable.

Correva come non aveva mai corso, come nessuno aveva mai corso (comme aucun être humain n'avait couru? comme un cheval, comme un ange?). "Comme personne n'avait jamais couru " précise la troisième et dernière version du roman (chap. XIII, p. 154) ${ }^{20}$.

Cette course est absolument unique et de fait peut-être devient-elle par là même exemplaire. Elle débouche finalement sur un bois où les arbres font mur. "Poi gli si parò davanti un bosco e Milton vi puntò dritto. Come entrò sotto gli alberi, questi parvero serrare e far muro e a un metro da quel muro crollò.» (chap. XIII, p. 155). Terme extrêmement prégnant que ce «bois", de par sa signification allégorique mais dont nous pouvons assurément penser qu'il est un lieu obscur où à la fois l'on se perd et l'on va à la rencontre d'une révélation ${ }^{21}$.

Peut-être Milton accomplit-il la moitié du nom qu'il s'est choisi. Ses yeux sont aveugles: il voit très peu de la terre et rien du ciel. Il n'y a pas de paradis à reconquérir. Tout au plus peut-être répond-il à la signification de la signature cryptée de Fulvia telle qu'elle nous est indiquée au début du roman.

$$
\begin{array}{l|l}
\mathrm{Fu} & 1 \\
\hline \mathrm{vi} & \mathrm{a}
\end{array}
$$

Signature que l'on peut décomposer, comme le suggère une étude, en $F u$ illla via ${ }^{22}$. Mais notre hypothèse, eu égard à ce qui a été dit précédemment, est que c'est Milton lui-même qui, à l’origine, a suggéré à Fulvia, ce cryptage.

20. Dans la première version il est écrit seulement "correva, correva". La deuxième précise "correva come non aveva mai corso ". Voir à ce propos l'édition annotée par Maria Antonietta Grignani, : Beppe Fenoglio Opere, a c. di M. Corti, M. A. Grignani, Einaudi, 1978, p. 2263-2273.

21. Le bois nous semble avoir la même fonction symbolique que le désert dans d'autres textes (la fin de Teorema de Pasolini, par exemple). Un lieu où le sujet va à la rencontre de sa réalité profonde, dans une totale solitude.

22. Voir l'analyse de Maria Grazia Di Paolo, «La Fulvia fenogliana" in Beppe Fenoglio, fra tema e simbolo, Ravenna, Longo edit., 1988, p. 52: «Fulvia, oltre a rappresentare l'eterno femminino a lungo accarezzato e disperatamente cercato assume un ruolo altamente positivo, quale ispiratrice della sua attività scrittoria [...]. Fu il/la via colei che spinge Fenoglio-Milton a scrivere». 
Il y a un décalage entre l'apparente lisibilité de ce roman et la difficulté à le cerner, en le mettant en perspective avec d'autres romans de Fenoglio. Roman d'un amour idéalisé destiné à ne jamais se réaliser, histoire d'une déclaration d'amour destinée à ne jamais pouvoir se dire vraiment si ce n'est au seuil de la mort, ce roman semble pouvoir se lire comme le roman d'apprentissage d'une désillusion, comme une fable sur l'écroulement de toute illusion.

Quel que soit le sens à tirer de cette "questione privata», elle met de toute façon en évidence, en contrepoint, la réalité de la violence de la guerre, la crudité, l'obscénité des corps mis à mal dans la guerre. (Voir à ce propos la description sanglante de la bataille de Verduno et l'épisode de la tonsure de l'institutrice fasciste, chap. XI).

La question personnelle qui semble, dans un premier temps, détourner le personnage de son implication dans la lutte, l'y reconduit dans l'écroulement final. Par un chemin dévié et qui suit un parcours très sinueux, elle redéfinit chez le partisan cet «être pour la mort» qui marque la catégorie absolue à laquelle appartiennent les combattants fenogliens. "La vita del partigiano è tutta e solo fatta di casi estremi [....] partigiano come poeta è parola assoluta, rigettante ogni gradualità" est-il dit dans $I l$ partigiano

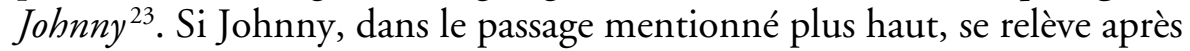
s'être écroulé, c'est pour s'écrouler définitivement, un peu plus tard dans une autre ellipse du verbe mourir ${ }^{24}$. Fenoglio, lui, qui a survécu de l'autre côté du mur, se relève pour se consacrer à l'ascèse de l'écriture. Mais seul celui qui meurt accomplit jusqu'au bout l'expérience qu'il a traversée.

On a parlé a propos de la fin de Il partigiano Johnny, d'une mort nécessaire, de la mort d'un héros qui, malgré le caractère juste de sa lutte, ne peut trouver au bout du compte que sa propre fin ${ }^{25}$. Je soulignerai ce verbe trouver, applicable à la quaestio personnelle de Milton, comme Johnny autre alter ego de Fenoglio, qui "poursuivant pour poursuivre autre chose encore» lui non plus ne peut trouver autre chose que sa propre fin $^{26}$.

23. Il partigiano Johnny, op. cit., p. 447.

24. "Johnny si alzò col fucile di Tarzan ed il semiautomatico... Due mesi dopo la guerra era finita." Ibid., p. 863.

25. Alberto Casadei, «Il sentimento della morte nel Partigiano Johnny e nella narrativa della resistenza italiana» in Transalpina, 2001, Vol. V, p. 114: «Fenoglio riesce a trovare un senso per la vicenda di Johnny soltanto nel suo non - senso, e il finale, così rapido e insieme così tragico, conferma la duplicità della vicenda stessa, epica ma inutile. Dunque, il finale del $P J$ parla di una morte necessaria, della morte di un eroe che, nonostante la giustezza della sua lotta, non può trovare altro che la propria fine».

26. Italo Calvino, op. cit. Voir note 1 de cette étude. 